\title{
BMJ Open Experiences engaging in a group-based physiotherapist-led exercise programme for adults living with HIV and complex multimorbidity: a qualitative study
}

\author{
Tina S. Homayouni, ${ }^{1}$ Alex Ruth, ${ }^{1}$ Zoe Abbott-Tate, ${ }^{1}$ Helen Burger, ${ }^{1}$ Shaera Rahim, ${ }^{1}$ \\ Carolann Murray, ${ }^{2}$ Ryan Wannamaker, ${ }^{2}$ Soo Chan Carusone (I) ,,3 \\ Kelly K. O'Brien (iD) ${ }^{1,4,5}$
}

To cite: Homayouni TS, Ruth A, Abbott-Tate Z, et al. Experiences engaging in a group-based physiotherapistled exercise programme for adults living with HIV and complex multimorbidity: a qualitative study. BMJ Open 2021;11:e045158. doi:10.1136/ bmjopen-2020-045158

- Prepublication history and additional online supplemental material for this paper are available online. To view these files, please visit the journal online (http://dx.doi.org/10. 1136/bmjopen-2020-045158).

TSH and AR contributed equally.

TSH and AR are joint first authors.

SCC and KKO are joint senior authors.

Received 24 September 2020 Accepted 27 May 2021

Check for updates

(C) Author(s) (or their employer(s)) 2021. Re-use permitted under CC BY-NC. No commercial re-use. See rights and permissions. Published by BMJ.

For numbered affiliations see end of article.

Correspondence to

Dr Kelly K. O'Brien;

kelly.obrien@utoronto.ca

\section{ABSTRACT}

Objective To explore experiences participating in a group-based physiotherapist (PT)-led exercise programme among people living with HIV and complex multimorbidity.

Design We conducted a qualitative descriptive study using semistructured interviews.

Recruitment and setting We recruited communitydwelling adults living with HIV who engaged in a groupbased PT-led exercise programme within an HIV-specialty hospital in Toronto, Canada. Interviews were conducted in-person or by telephone.

Participants Eight men and two women with a median age of 58 years and median of six concurrent conditions in addition to HIV, who had attended $\geq 2$ classes of the exercise programme.

Data collection Interviews explored (1) reasons for engaging in the programme, (2) experiences with exercise prior to and after joining the programme, (3) facilitators and barriers to engagement and (4) perceived impacts of participation on health and disability. We administered the HIV Disability Questionnaire and a demographic questionnaire.

Results Experiences spanned perspectives prior to, during and after the PT-led exercise programme. Reasons for engaging in the programme included addressing health-related goals. Participants identified accessibility, the flexible schedule, interprofessional staff and the HIV-specific, group-based environment as facilitators to engagement. Participants reported high attendance rates, but identified episodic health challenges and overcrowded space as potential barriers to attending exercise classes. Perceived impacts on health and disability outcomes included improved physical, mental, social and cognitive health, and activities of daily living. Anticipated or actual experiences transitioning to independent exercise included facilitators (supportive programme leaders) and barriers (challenges motivatiing self to exercise alone).

Conclusions Features of the programme that facilitated engagement included the interprofessional, group-based environment that offered tailored exercise in an HIVspecific facility, whereby participants perceived benefits in domains of health and disability. However, challenges transitioning to independent exercise remain. Group-based PT-led exercise programmes may facilitate engagement
Strengths and limitations of this study

- To our knowledge, this is the first qualitative study to explore experiences of people living with HIV and complex multimorbidity in a group-based physiotherapist-led exercise programme in Canada.

- Insights gained from this study can inform future exercise programming for people living with HIV and provide support for successful transition to independent exercise.

- The exercise programme in this study was offered in an urban setting in a high-income country at an HIV-specialty hospital, therefore, replicating environmental and programme characteristics may be challenging in rural settings with fewer resources.

- Participants were mainly community-dwelling White men, living with HIV and complex multimorbidity, hence findings may not be transferable to broader diverse populations of people living with HIV.

in exercise among adults living with HIV and complex multimorbidity.

\section{INTRODUCTION}

People living with HIV (PLWH) are living longer, but are susceptible to multimorbidity resulting from HIV, ageing and side effects from medications. ${ }^{1-5}$ Multimorbidity, defined as living with two or more concurrent health conditions in addition to HIV, ${ }^{6-8}$ can result in physical, cognitive, mental and social health challenges and uncertainty about future health, collectively referred to as disability, ${ }^{910}$ which may be exacerbated by extrinsic (stigma) and intrinsic (older age) factors. ${ }^{11}$ Hence, it is important to consider rehabilitation interventions to address disability and contextual factors experienced by PLWH.

Exercise is a self-management intervention that can mitigate disability in PLWH. ${ }^{12} 13$ 
Exercise is safe and can improve physical fitness and mental health in adults living with HIV. ${ }^{14-16}$ Despite this, PLWH often have sedentary lifestyles and are more physically inactive than other populations with chronic diseases. ${ }^{17} 18$ Barriers to PLWH engaging in exercise can include lack of knowledge about exercise, mental or physical illness, time constraints, financial inaccessibility to gym environments, multimorbidity and stigma. ${ }^{19-21}$ PLWH may experience greater barriers to exercise if they have lower socioeconomic status or mental health challenges compounding their multimorbidity, highlighting the complexity of this population. ${ }^{22} 23$ Hence, PLWH with complex multimorbidity may benefit from tailored exercise programmes that consider this complexity and address barriers to exercise.

Community-based exercise programmes (CBEPs) can enhance exercise engagement among PLWH. ${ }^{24}$ CBEPs involve a group of people with similar exercise considerations or health conditions, with a goal of safely transitioning to independent exercise. ${ }^{25}{ }^{26}$ CBEPs currently exist for a range of chronic and episodic conditions including stroke, multiple sclerosis and HIV. ${ }^{24-27}$ In a 16-week supervised CBE intervention, PLWH described positive experiences with an accessible and nonjudgemental gym environment, and positive changes in physical, social and mental health. ${ }^{24}$ However, the episodic nature of HIV and multimorbidity influenced exercise engagement. ${ }^{24}$ Similarly, PLWH identified uncertainty and complexity of HIV and multimorbidity as factors that influenced their readiness to engage in exercise.$^{20}$ Hence, CBEPs should consider the complexity and episodic nature of HIV to maximise engagement in exercise. ${ }^{111718}$

Physiotherapists (PTs) have knowledge of diseasespecific exercise physiology and can tailor exercise to account for the episodic nature of HIV and multimorbidity. ${ }^{28}{ }^{29}$ PTs play a role in HIV care addressing physical, psychological and social health while considering contextual domains of multimorbidity, episodic nature of HIV, ageing, stigma, resource security, continuity of care, competing priorities and social isolation. ${ }^{30}$ Thus, PTs can help address barriers to exercise engagement among PLWH with complex needs. Three known PT-led exercise programmes for community-dwelling PLWH in the UK, Hong Kong and South Africa demonstrated promising results with improvement in physical and emotional health, quality of life and activity limitations. ${ }^{28} 3132$ However, the extent of multimorbidity and experiences engaging in these programmes with differing healthcare systems among PLWH is unclear. ${ }^{28} 313334$ Our aim was to explore experiences engaging in a 12 week group-based, PT-led exercise programme from the perspective of adults living with HIV and complex multimorbidity. Specific objectives were to explore: (1) reasons for engaging in the programme, (2) perceived facilitators and barriers to participation, (3) levels of engagement and (4) perceived changes and impact of participation on health and disability outcomes.

\section{METHODS}

\section{Study design}

We conducted a qualitative descriptive study using semistructured interviews to explore the experiences of PLWH who participated in a group-based PT-led exercise programme. $^{35}$

\section{Setting and exercise program}

Launched in 2019, the PT-led exercise programme is part of a publicly funded day health programme for PLWH and multimorbidity at Casey House, an HIV-specialty hospital in Toronto, Canada. ${ }^{36}$ The goal of the day health programme is to increase access to health-related services for PLWH and improve health and quality of life. ${ }^{36}$ The cost-free programme includes a range of interprofessional services including physiotherapy, social work, massage therapy, recreation therapy and nursing. ${ }^{36}$

The exercise programme is a 12-week programme involving group-based exercise classes. Sessions are supervised by a PT and registered nurse (RN) who are responsible for determining eligibility, assessment and enrolment. Exercise classes occur three times per week; clients are advised to attend two classes per week. Each 60 min class includes a warm-up, circuit training and cool down. Eleven circuit stations involving $60 \mathrm{~s}$ of aerobic, resistance and balance exercises are completed twice by participants. The gym is equipped with modern exercise equipment including weights, treadmills and an arm ergometer. Clients are monitored throughout the class using the Borg Scale of Perceived Exertion and additional safety measures (eg, blood pressure) as required. ${ }^{37}$ After completing twelve weeks, clients requiring further support can enrol in the weekly maintenance exercise programme.

\section{Patient and public involvement}

This research evolved from a long-standing communityacademic-clinical partnership among people ageing with HIV, researchers, and clinicians who identified key research priorities in HIV, ageing and rehabilitation as part of the Canada-International HIV and Rehabilitation Research Collaborative (CIHRRC). ${ }^{38} 39$ CIHRRC conducted an international multistakeholder consultation to establish a Framework of Research Priorities in HIV, Ageing and Rehabilitation. ${ }^{39}$ This research addresses three research priorities identified in this Framework, specifically examining (1) strategies for chronic disease management and healthy ageing with HIV; (2) facilitators and barriers to access and engagement in, rehabilitation and (3) effectiveness of rehabilitation interventions for healthy ageing with HIV. ${ }^{39}$

We consulted with a community member living with HIV familiar with the exercise programme who advised on the development of the data collection tools and participated in pilot interview with the team. Results from this study were translated in the form of a poster summary shared with study participants, and a presentation with Casey House staff. Results from this study informed the 
ongoing development of the role of physiotherapy and implementation of a PT-led exercise programme in the interprofessional HIV care setting for people living with complex multimorbidity. ${ }^{40}$

\section{Participants}

We recruited community-dwelling adults (18 years of age or older) living with HIV who had attended two or more classes of the exercise programme using posters and word of mouth via staff at Casey House. We used purposive sampling, ${ }^{41}$ with the aim of recruiting a sample with two or more women and at least two participants who had completed the 12-week programme. Members of the team (TSH, AR, ZA-T, HB and SR) communicated with interested participants by phone or email to confirm eligibility, review study objectives, schedule an interview and obtain verbal or written consent to participate in the study. We obtained written consent from participants for in-person interviews, and verbal consent (confirmed in writing by the researcher) for telephone interviews.

\section{Data collection}

Between January and April 2020, five team members (TSH, AR, ZA-T, HB and SR) interviewed participants face to face or by telephone. Interviews were $60-90 \mathrm{~min}$ in duration. Using a semistructured interview guide, we asked participants about (1) their experiences with exercise prior to and after joining the programme, (2) reasons for engaging in the programme, (3) facilitators and barriers to engagement and (4) perceived impacts of participation on health and disability (online supplemental file 1). During the preliminary interviews, participants described specific behaviours and factors related to the exercise programme, which we believed may be relevant to future interviewees. Thus, we revised our interview guide three times to include specific questions and prompts related to these new themes and to refine our questions. Field notes were taken during the interviews to capture context and aid in data analysis, using methodology described by Phillippi and Lauderdale. ${ }^{42}$

We administered a self-reported demographic questionnaire to describe personal characteristics including, age, ethnicity, source of income, gender, year of HIV diagnosis, viral load, number of concurrent health conditions, travel time to the programme, and exercise/physical activity levels prior to initiating the programme. Also, we administered a self-reported disability measure called the HIV Disability Questionnaire (HDQ) to describe disability experienced by the participants. The HDQ is a 69 -item self-reported questionnaire that describes the presence, severity and episodic nature of six domains of disability for PLWH. ${ }^{43}$ The HDQ is a reliable and valid tool to measure disability in PLWH. ${ }^{44-47}$ Our aim for using the HDQ in this study was to describe characteristics of disability experienced by participants at the time of the interview, and was not included in our qualitative interview analysis, but rather provides context to describe the overall health and disability among the sample population.

\section{Data analysis}

Interviews were audiorecorded, transcribed verbatim and reviewed for accuracy. We conducted a qualitative descriptive analysis of interview transcripts and field notes. ${ }^{48} 49$ We conducted a constant comparison analysis whereby data collection and analysis occurred concurrently. ${ }^{41}$ All team members independently read and coded, line by line, the first two transcripts to create codes related to our study objectives and develop a preliminary coding scheme. Codes were organised into broader themes related to our research objectives. All team members independently coded a third transcript referring to the coding scheme, adding new codes to the scheme as they emerged. Two team members independently coded the remaining transcripts and discussed new codes with the team. We determined our final study sample size based our ability to address our study objectives. Reaching thematic saturation was not a goal of our study. Our aim was to obtain meaningful and applicable information through the exploration of the themes generated during the interviews, which could then be used to inform future programming for PLWH and complex multimorbidity. We developed participant summaries for each interview that included participant demographic information and a summary of the interview in relation to our study objectives. We used NVivo software (QRS International, NVivo, V.12) to organise our data.

We calculated the median and 25-75th percentiles of the HDQ presence, severity and episodic scores by summing the responses in each domain and transforming them to a score out of 100 . Higher scores indicate greater presence, severity and episodic nature of disability. ${ }^{50} \mathrm{We}$ calculated the median and 25-75th percentiles for continuous data, and frequency and percentages for categorical variables in the demographic questionnaire. ${ }^{51}$

\section{RESULTS}

All 10 PLWH who expressed interest, participated in a face-to-face $(n=5)$ or telephone $(n=5)$ interview between January and April 2020. The majority of participants were men, median age of 58 years, and a median of six self-reported concurrent health conditions in addition to HIV (table 1). The highest median HDQ severity and presence scores were in the mental-emotional domain (online supplemental file 2). The lowest median HDQ severity and presence scores were in the difficulties with day-to-day activities domain (online supplemental file 2). All participants scored a median of zero in the episodic domain that describes the extent of daily fluctuations in disability (online supplemental file 2). At the time of interview, five participants were enrolled in the core 12-week exercise programme and five in the weekly maintenance programme. The majority of participants $(n=6)$ reported regularly engaging in physical activity (any bodily movement produced by skeletal muscles resulting in energy expenditure), ${ }^{52}$ most commonly walking. 
Table 1 Characteristics of participants $(n=10)$

\begin{tabular}{|c|c|}
\hline Demographic characteristics & No of participants (\%) \\
\hline Median age (years) (25th, 75th percentile) & 58 years $(53,68)$ \\
\hline \multicolumn{2}{|l|}{ Gender } \\
\hline Man & $8(80)$ \\
\hline Woman & $2(20)$ \\
\hline \multicolumn{2}{|l|}{ Ethnicity } \\
\hline Aboriginal, First Nations, Métis or Inuit & 1 \\
\hline African, Caribbean or black & 1 \\
\hline White & 7 \\
\hline South Asian & 1 \\
\hline $\begin{array}{l}\text { Median year of HIV diagnosis } \\
\text { (25th, } 75 \text { th percentile) }\end{array}$ & $1994(1988,2000)$ \\
\hline Currently taking antiretroviral medication & $9(90)$ \\
\hline $\begin{array}{l}\text { Undetectable viral load ( }<50 \text { copies } / \mathrm{mL} \text { of } \\
\text { blood) }\end{array}$ & $9(90)$ \\
\hline $\begin{array}{l}\text { Median number of concurrent health } \\
\text { conditions, in addition to HIV ( } 25 \text { th, } \\
\text { 75th percentile) (Range of concurrent } \\
\text { conditions) }\end{array}$ & $6(4,10)(2-13)$ \\
\hline \multicolumn{2}{|l|}{$\begin{array}{l}\text { Most common self-reported concurrent } \\
\text { health conditions ( } \geq 20 \% \text { of sample) }\end{array}$} \\
\hline $\begin{array}{l}\text { Mental health conditions (anxiety, } \\
\text { depression and other) }\end{array}$ & $7(70)$ \\
\hline Cardiovascular conditions & $6(60)$ \\
\hline Muscle pain & $5(50)$ \\
\hline Bone and joint disorders & $4(40)$ \\
\hline Eye disorders & $3(30)$ \\
\hline Diabetes & $2(20)$ \\
\hline \multicolumn{2}{|l|}{ Primary income source } \\
\hline $\begin{array}{l}\text { Government disability support } \\
\text { programmes }\end{array}$ & $5(50)$ \\
\hline Pension & $4(40)$ \\
\hline Employment & $1(10)$ \\
\hline $\begin{array}{l}\text { Median travel time to exercise programme } \\
\text { (minutes) (25th, } 75 \text { th percentile) }\end{array}$ & $11(5,40)$ \\
\hline $\begin{array}{l}\text { Exercising independently (outside the PT- } \\
\text { led exercise programme) }\end{array}$ & $3(30)$ \\
\hline \multicolumn{2}{|l|}{$\begin{array}{l}\text { Self-described exercise levels (including } \\
\text { within the PT-led exercise programme) }\end{array}$} \\
\hline $\begin{array}{l}\text { I currently exercise regularly but I only } \\
\text { began exercising within the last } 6 \\
\text { months }\end{array}$ & $2(20)$ \\
\hline $\begin{array}{l}\text { I currently exercise regularly and I have } \\
\text { been exercising longer than } 6 \text { months }\end{array}$ & $4(40)$ \\
\hline I currently exercise but not regularly & $2(20)$ \\
\hline $\begin{array}{l}\text { I currently do not exercise but I am } \\
\text { thinking about it in the next } 6 \text { months }\end{array}$ & $2(20)^{*}$ \\
\hline
\end{tabular}

*One participant selected this option on the demographic questionnaire but stated, during the interview, that they were still exercising in the maintenance programme once a week.

PT, physiotherapist.

Participants'experiences spanned perspectives prior-to engaging in the program, during, andafter the program. Experiences included: (A) reasons for engaging in theprogram, (B) factors influencing engagement, (C) perceived impacts of participationon health and disability, and (D) anticipated or actual experiencestransitioning to independent exercise (figure 1).

\section{A) Reasons for engaging in the group-based PT-led exercise programme}

Participants perceived barriers to exercising in traditional gym environments, which made the group-based PT-led exercise programme in a community hospital setting more appealing. Personal goals for engaging in the exercise programme were also discussed.

\section{Barriers to traditional exercise and environments}

Participants described extrinsic barriers to exercise such as the physical and financial inaccessibility of traditional gyms and stigma in the community.

I'm on [government funding for disability] so it's very hard to live with the funds that we have. [...] so the check just goes towards food and rent so I can't afford a gym membership. (P4)

Intrinsic barriers were also identified, including discomfort in conventional gyms, body image concerns, lack of safe exercise knowledge, social isolation, lack of motivation to exercise and physical limitations.

[At a traditional gym] you're embarrassed about your body type, or you're embarrassed about some kind of physical thing, or whatever, here [...] I don't feel I'm being judged at all in that department. So...I'm willing to continue doing it. (P3)

Barriers influenced participants' previous levels of exercise and physical activity.

\section{Goals of engagement}

Participants described health-related goals for engaging in the programme. Most frequently reported were physical health goals, followed by mental-emotional health goals, such as managing anxiety and depression. Social health goals, such as meeting new people, were also described. Physical health goals included managing concurrent health conditions, remaining mobile and independent with age, and improving fitness:

I'm close to being sixty [...] I want to remain like, healthy and active...So... I don't want to start to lose like, muscle mass and have like, ... bad posture, and not being able to use my leg anymore. All of those things. So, ...I just want to- to remain active and healthy for as long as I can. (P7)

\section{B) Factors influencing engagement in the group-based PT-led exercise programme}

Factors that positively influenced participants' engagement in the programme included financial and physical accessibility, the welcoming and supportive group environment, safety and supervision, tailored exercise and flexibility with episodic health. 


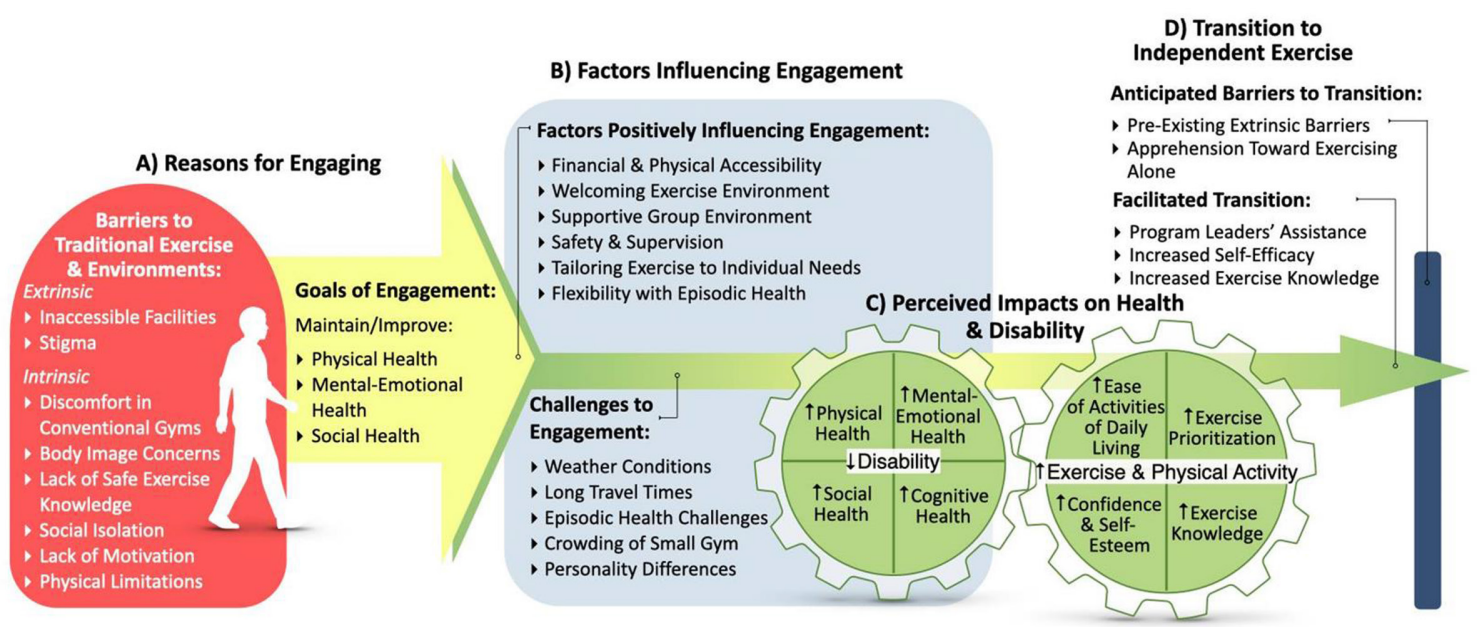

Figure 1 Overview of experiences engaging in a group-based PT-led exercise programme among adults living with HIV and complex multimorbidity. PT, physiotherapist.

\section{Financial and physical accessibility}

Many participants were previously accessing day health programme services, allowing easy referral to the exercise programme. The programme was cost-free and the building physically accessible, both of which facilitated attendance. The majority of participants lived within walking distance of the facility:

It was close by, I live in the neighborhood so that was number one, [...] because of the neighborhood, I mean it's $[\ldots]$ a gay-friendly neighborhood and $[\ldots]$ it was accessible, easily accessible. (P6)

\section{Welcoming exercise environment}

Participants described the group-based PT-led exercise programme, hosted in an HIV-specific institution, as welcoming and safe, with accessible staff and peers with HIV-specific knowledge:

Everyone was so welcoming and supportive and kind and generous [...] I have nothing but good things to say about it." (P6)

While participants found the exercises challenging, they enjoyed the high-energy environment with music:

You don't realize you're exercising cause you're having such a good time. (P3)

\section{Supportive group environment}

Participants described the group as supportive and nonjudgemental, contributing to a positive experience. Being among a group of PLWH was comforting as participants felt they could talk openly without fear of stigma and without worrying about judgement from others:

Here you don't have to worry about your image. When you're pulling off your shirt and your stomach is sticking out [...] That'd be a little bit different when you're at [a community centre]. (P1)
Participants were able to learn from other group members due to differences in experiences, fitness level and disability. Despite this diversity, several participants used the expression of being 'in the same boat' with similar experiences living with HIV. Participants found the group reinforcing and motivating:

It's surprising how it can motivate you when you're with other people... look at this fella, he's not as, uh, you know healthy as you are but look how much energy he's putting into it. It gives you that boost to push a little harder, you know, it's nice to be in a group. It's surprisingly beneficial but you don't even realize it at first. (P8)

\section{Safety and supervision}

Participants reported positive experiences with the safety and supervision that they received from the interprofessional (PT and RN) programme leaders, whom they described as 'positive' and 'inspirational':

[...] they're very... positive and they give people affirmations $[\ldots]$ giving your moral support you know, getting you to go a little more you know each time you progress. (P8)

Many participants mentioned they felt safe while supervised by healthcare professionals, including one participant who was apprehensive about exercising before joining the programme due to a history of cardiac issues:

The fact that I knew [...] I was being supervised, and it was monitored, and um, these were professionals and they knew what they were doing, gave me the confidence to participate. [...] Having somebody who knows what they're doing show you how to exercise properly without injuring yourself, and how to modify an exercise so that you can get to a certain level where you can actually do it. (P3) 


\section{Tailoring to individual needs}

Participants positively reflected on how the PT modified exercises during the classes, tailoring to their unique needs and levels of ability. Participants with specific concerns or goals received take-home exercises:

Each exercise had a component where you could $[\ldots]$ make it $[\ldots]$ more challenging or less challenging. You could start off at a certain level if you are very weak in an area, but then other people could up the ante of the same exercise [...] there was always sort of an adaptation to it $[\ldots]$ to each exercise that fit the person. (P6)

\section{Flexibility with episodic health}

Participants described the programme as 'flexible'. Those experiencing episodes of disability were readmitted from maintenance to the core programme. Furthermore, participants appreciated opportunities to supplement classes that they missed when unwell:

[...] the thing with HIV, you never know what you're going to feel like in the day you know whether your energy's low or if you know pain's gonna keep you up through the night $[\ldots]$ they understand and accept that you know there's days that you just can't make it but they'll fill you in in another class which is great. (P3)

\section{Challenges to engagement}

While participants easily identified numerous facilitators to attending the exercise programme, challenges to engagement and attendance were less commonly described, and required more probing questions from the interviewer. Participants reflected on factors that challenged engagement, and most commonly identified poor weather conditions and travel times, which could compound on one another.

$[\ldots]$ by the time you know you walk through the ... the snow storm, you know five blocks to get to the bus stop, and then you get on the bus in the subway and then you walk four blocks to get there it's just a little difficult sometimes when you're not, you know, at $100 \%$ [...] health. (P8)

Some participants identified challenges related to differences in personalities causing friction, or occasional overcrowding:

[...] we've got a small gym here, and when there's too many people it makes it a little bit uncomfortable.

\section{C) Perceived impacts of the exercise programme}

All participants perceived that engagement in the groupbased PT-led exercise programme positively influenced their perceived level of disability in one or more domains (physical, mental-emotional, social, cognitive and activities of daily living). Participants reported increased physical activity and greater ability to engage in exercise.

\section{Physical health impacts}

Participants reported improved physical health, describing increases in strength, endurance and balance:

$[\ldots]$ instead of like, taking the elevators, I would take the stairs. Because I felt like I- I was ... building up endurance, $[\ldots]$ I felt like I was in better shape. (P7)

Participants also described how participation in the program helped manage physical symptoms of their health conditions, primarily pain and fatigue:

$[\ldots]$ on the bike, walking on the machine... That is helping me. Now, I don't feel much pain, and I don't take, everyday, ... my pain killers. I don't take my pain killers; I take one- when I feel pain [...] But not every day. So, I have seen things are changing. (P9)

Perceived physical improvements that participants attributed to the exercise programme subsequently facilitated activities of daily living.

\section{Mental health impacts}

All participants perceived that participation in the programme positively influenced their mental health, by helping manage mental-emotional symptoms, or contributing to a positive mood overall:

Before [the class], not normal person, I have depression, I have, uh I can't sleep, [...] Now is I'm normal, I'm a strong person, I'm a healthy person. (P10)

Participants reported improved confidence and selfesteem and less fear around exercising. Three participants described a sense of accomplishment from achieving gains in physical fitness, which increased their motivation to exercise:

Before the exercise, I thought I was going to die. [...] Now, we have exercise, makes me stronger, makes me $[\ldots]$ want to do more and more. (P5)

\section{Social impacts}

Participants described increased social inclusion as they met new people and developed friendships. They emphasised the importance of being a part of the community, as many had felt socially isolated in the past:

[...] I feel accepted, I feel warm inside, it's giving me a feeling of community, and acceptance that I was kind of missing in my life. You know I don't have a lot of friends, so you know it's nice to do it as a group of people, when I walk in say 'hi, how are you?' and it's a wonderful feeling, it's the community in and of itself. (P8)

Participants felt social inclusion from the supportive group was compounded by improvements in other health 
domains and motivated them to seek social opportunities outside the programme.

I think I'm doing more things now than I ever did before, because my health has improved, and I'm more self-confident. [...] Now, I'm more willing to go see a movie or to go out for dinner, or if I'm invited out to somebody's place. But before I was just not- did not feel well enough to do that. [...] I'm not so housebound. Which is good for me. And I'm willing to, you know, expand my horizons socially. (P3)

\section{Cognitive impacts}

Three participants perceived cognitive improvements, including greater clarity of thought and improved concentration:

I think the more you exercise, I think the sharper your mind becomes. For me anyways, 'cause I could just, it just helps, it just clears my mind. (P3)

Overall, participants described an interconnectedness of physical, mental and social health, where improvements in one domain often contributed to improvements in another.

[...] I was in pain and I needed help, and then Casey House was so friendly and on point in helping me with those [things], and the psychological and physical fitness came you know sort of afterwards [...] it all became one [...] I just got more motivated, more myself, at ease and more comfortable and welcomed $[\ldots]$ then I realized that what I was doing for my mind and for my body and in general so those were all... they all sort of piggy-backed off one another. (P6)

\section{Exercise and physical activity levels}

Participants reported increases in exercise and physical activity levels. Outside the programme, most participants reported having increased their physical activity levels by either being more active at home, walking more, or taking the stairs over an elevator. Participants reported increases in exercise knowledge, self-esteem and prioritisation of exercise:

I have what it takes. I can do it. You know. So, um, sobut I needed [the program] to kind of like confirm that for me.[...] [Exercise] has to be an essential part of my life, now. (P7)

Five participants said they rely on the program as their only form of structured exercise. The other five participants reported exercising at home, or at community centers in addition to the program.

\section{D) Perceptions of the transition to independent exercise}

The group-based PT-led exercise programme strives to prepare clients for transition to independent exercise, defined as exercising regularly in the community, individually or with peers, on completion of the exercise programme, or in combination with the maintenance programme. Of the five participants enrolled in the maintenance programme at the time of their interview, three reported not exercising independently. Participants discussed anticipated or actual experiences transitioning to independent exercise, which included programme features that facilitated the transition, as well as anticipated concerns or barriers to independent exercise.

\section{Facilitated transition}

Participants reported that the programme leaders actively encouraged them to strive towards independent exercise, helping to find accessible gyms, and attempting to decrease reliance on the programme. Participants expressed feeling more confident and knowledgeable about safe exercise technique, and were motivated to continue exercising after the programme due to improved self-efficacy:

It's not so- so uh, so daunting anymore. Uh, and- and I can see myself, like, uh, after [the program], to move on to a place where I can, uh, do it somewhere else. [...] I did not even contemplate that idea before. [...] I developed self-confidence about the fact that, yes, I'm ok, I'm able to do it. (P7)

Three of the five participants interviewed after the programme ceased due to COVID-19 reported exercising at home, using exercises learnt in the programme or online resources.

\section{Anticipated barriers to successful transition}

Participants stated that some barriers to exercise perceived prior to programme enrolment will make for a difficult transition to independent exercise. Commonly reported concerns included lack of motivation to exercise alone, financial barriers, and discomfort in public gyms:

The social anxiety of going to a gym or even to a gym at my building, umm, I guess the judgement, whereas here it didn't feel that way. (P4)

Two participants interviewed following the programme cessation due to COVID-19 struggled with motivation:

[...] if I do it on my own, it's not the same [...] if I'm looking at, uh, at an instructor online, it might give me the motivation that I need [...] if there's no instructors there, or whatever, [...] I'm not even doing anything. (P7)

\section{DISCUSSION}

To our knowledge, this is the first qualitative study exploring experiences engaging in a group-based PT-led HIV-specific exercise programme from the perspective of PLWH and complex multimorbidity in Canada. Prior to engaging in the programme, participants reported having health and fitness-related goals but faced barriers to exercise. The exercise programme helped mitigate 
these barriers due to the interprofessional and HIVspecific environment, flexibility and ability to influence perceived disability. However, participants described concerns and difficulty transitioning to independent exercise, attributed to anticipated barriers exercising in the broader community and remaining motivated when no longer in a structured and supervised programme.

\section{Reasons for engaging in the programme}

Goals related to improving health was a major reason for engaging in the exercise programme among participants. Collaborative goal setting between PTs and PLWH can increase PLWH's sense of control towards their rehabilitation and exercise ${ }^{53}$ which can translate to increased adherence to self-management strategies and consequently, physical activity. ${ }^{54-56}$ Goals should be concrete, collaborative, realistic and measurable for optimal goal attainment and behaviour change as seen in adults with chronic pain. ${ }^{57}$ The Goal Attainment Scale is a beneficial rehabilitation tool for the creation of salient and concrete goals, and was used to quantify personal exercise goals throughout an exercise programme for PLWH in the UK. ${ }^{285859}$ Future exercise programmes for PLWH should employ goal setting frameworks to increase adherence to exercise and physical activity.

\section{Environment in an HIV-specific day health program}

The zero-cost access to the programme, its HIV-specific design and structure, and its co-location within a multidisciplinary day health programme facilitated engagement. A PT programme in a multidisciplinary setting may facilitate adherence to rehabilitation as PLWH can access multiple programmes addressing disability. ${ }^{60} 61$ Additionally, financial and physical accessibility is a motivator to engage in CBEPs for PLWH. ${ }^{24}$ As participants faced complexity due to multimorbidity, age, frailty and financial burdens, accessibility was paramount when promoting exercise engagement. HIV-specific CBEPs can consider these complexities to facilitate attendance to exercise programmes. ${ }^{192021}$ As people living with stroke and chronic pain report similar barriers to exercise as PLWH, CBEPs for chronic conditions (including HIV) can adopt similar attributes to increase exercise engagement. ${ }^{19} 266263$ Stigma can impact willingness to engage in CBEP, which for some can result in preferences for an HIV-specific programme, while others may prefer non-HIV specific programmes that limit HIV disclosure. The HIV-specific nature of the exercise programme was an important component identified in our study, as HIV disclosure among participants who were uniquely complex allowed for a supportive group environment to which some participants attributed to having similar life experiences. Future programmes should consider the complexity of PLWH to determine the necessity of an HIV-specific structure, and implement an accessible programme with a multidisciplinary approach to rehabilitation to increase engagement.

\section{PTs as interprofessional leaders}

Participants appreciated the specialised interprofessional (PT and RN) team, as their HIV-specific knowledge, safety monitoring and tailored exercise facilitated engagement. Supervising personnel in exercise programmes for PLWH should understand complex health conditions that can present barriers to accessing and engaging in services. ${ }^{22} 3061$ RNs may be especially helpful when working with medically complex populations such as PLWH through symptom and medical management, selfcare education and maintaining continuity of care. ${ }^{64-66}$ PTs can also adopt approaches sensitive to complexities to mitigate these barriers, addressing physical, psychological and social challenges while considering contextual factors and the potential episodic nature of chronic conditions. ${ }^{22}{ }^{30}$ In a study exploring the readiness to engage in exercise, PLWH and multimorbidity felt apprehensive towards exercise due to risk of injury, ${ }^{20}$ but reported less safety concerns with guidance from interprofessional programme leaders. To further enhance exercise adherence and engagement in PLWH and complex multimorbidity, CBEPs may benefit from supervision by interprofessional teams of healthcare professionals who consider HIV-specific needs and complexities, and promote safety and exercise effectiveness.

\section{Episodic nature of HIV}

Participants reported experiencing fluctuations in health and disability, and the flexible nature of the exercise programme allowed continued participation and adherence to exercise. While participants scored low on the episodic subscale of the HDQ (online supplemental file 2), the HDQ specifically measures health fluctuations experienced during the past week. Therefore, the fluctuations in health described by our participants likely refer to experiences over a longer period of time. Furthermore, Montgomery et al reported that PLWH withdrew from a CBEP due to fluctuations in health. ${ }^{24}$ Participants in this study reported similar setbacks during the maintenance phase, resulting in temporary cessation of the programme; however, they were able to re-enrol in the thrice weekly class for additional exercise guidance. In another PT-led exercise programme, one-third of PLWH restarted as a result of its open-access structure, allowing continued engagement despite episodic health challenges. ${ }^{28}$ Hence, CBEPs for PLWH and complex multimorbidity should be flexible to accommodate fluctuations in health and promote continued exercise engagement.

\section{Perceived benefits of the exercise programme}

Participants perceived benefits in several domains of health that positively influenced one another. Perceived improvements in social health were attributed to the group-based nature of the exercise programme, as participants reported increased feelings of connectedness and social support, as well as 
a result of improvements in other domains of health. Studies evaluating the impacts of CBEPs for stroke survivors and people living multiple sclerosis found that improvements in physical health led to improvements in confidence and increased social participation outside the programmes. ${ }^{63} 67$ Social support is associated with increased health-related quality of life and protects against depression among PLWH, ${ }^{68-70}$ while lack of social support is a common barrier to exercise for PLWH and those living with chronic pain. ${ }^{62} 71$ Hence, group-based exercise programmes for PLWH can simultaneously influence mental health and facilitate exercise engagement. Systematic review evidence and qualitative studies have documented physical and mental health benefits among PLWH engaging in exercise. ${ }^{14-162428} 32607273$ Additionally, results from quantitative systematic reviews showed that exercise improved self-reported quality of life in cognitive domains, ${ }^{16} 73$ although a previous study using objective outcome measures indicated that a PT-led aerobic exercise programme did not improve cognition in PLWH. ${ }^{33}$ While three participants in our study felt perceived benefits in cognition, this is an area for future study.

\section{Anticipated barriers to transition}

Though the exploration of barriers to transitioning to independent exercise was not an objective of this study, this emerged as an important theme. Despite perceived benefits of the programme, the long-term uptake of exercise as a sustained source of behavioural change among PLWH and multimorbidity is unclear. Anticipated challenges to independent exercise included financial insecurity, fear of stigma and a lack of motivation to exercise alone, similarly reported by PLWH in previous studies as barriers to exercise..$^{20247174}$ Of the five participants in the maintenance programme, three reported relying on the weekly session as their only form of exercise; hence, some may remain in maintenance long term, which has implications for sustainability. The transtheoretical model of behavioural change suggests that maintaining a behaviour for 6 months is associated with increased confidence in continuing the behaviour long term, and less risk of relapse. ${ }^{75}$ Thus, a formal exercise programme of longer duration may be indicated in order to sustain change in exercise behaviour for PLWH. Application of behaviour change strategies such as self-monitoring, goal setting, education, scheduling and social support can be effective for increasing exercise and physical activity for PLWH, including those of low socioeconomic status. ${ }^{54}$ Future programming should consider implementing these behaviour change strategies when supporting participants in the transition to independent exercise.

\section{Exercise versus physical activity}

While the group-based PT-led exercise programme strived to prepare clients for transition to independent exercise, our findings suggest it may not be realistic for some PLWH and complex multimorbidity to independently exercise regularly due to perceived barriers and episodic health challenges. The Canadian Physical Activity Guidelines recommend $150 \mathrm{~min} /$ week of moderate to vigorous aerobic physical activity and muscle strengthening at least twice a week for adults. ${ }^{77}$ Exercise is defined as a structured subset of physical activity done to maintain or improve physical fitness, whereas physical activity is defined as any bodily movement produced by skeletal muscles resulting in energy expenditure. ${ }^{52}$ Vader et al reported that PLWH perceived 'physical activity' as any activities involving mobility and 'exercise' as structured activities to improve fitness. ${ }^{78}$ Some PLWH perceived 'exercise' to be more intimidating than 'physical activity' and authors reported that increasing physical activity may be a more realistic long-term goal. ${ }^{78}$ Furthermore, a study conducted with Mozambican women living with HIV showed activities such as dancing and active games can yield comparable health benefits to structured exercise. ${ }^{79}$ PLWH should strive to meet Physical Activity Guidelines, while allowing for flexibility due to the episodic nature of HIV, and may supplement regimented exercise with less formal physical activity to alleviate barriers. Clinicians should consider use of terminology such as 'physical activity' over 'exercise' to mitigate feelings of intimidation. Moreover, when developing an exercise programme and managing expectations, it is important to consider and clearly communicate the goals of the programme as increasing physical activity level, and/or preparing clients for transition to independent exercise.

\section{Implications for clinical practice and future research}

This study was completed as part of a larger mixedmethods study evaluating a PT model of care for PLWH and complex multimorbidity. ${ }^{80}$ Our results contribute to the understanding of experiences with the PT-led exercise programme in this model of care. Findings such as the importance of an interprofessional environment that provides social support and tailored interventions may help to inform broader CBEP striving to increase physical activity and exercise among PLWH. Contextual factors and the episodic nature of disability in PLWH should be considered by establishing accessible and flexible programming. Future studies should explore the costeffectiveness of PT-led exercise programmes for PLWH and multimorbidity, as well as experiences engaging in sustained physical activity following discharge from a formalised supervised exercise programme, to better determine the feasibility of broader scale up of exercise programming in the community, and sustainability of independent exercise for PLWH.

\section{Limitations}

The group-based PT-led programme is predominantly composed of men and is part of a publicly funded 
and highly supported interprofessional day health programme situated in a unique urban HIV-specialised hospital in Canada. ${ }^{36}$ Hence, experiences may not be transferable to other genders, or the broader population of PLWH including low to medium resource settings and without access to specialised programmes. The interruption of the exercise programme due to COVID-19 resulted in five interviews being conducted by telephone, hence body language could not be observed. However, this provided opportunities to explore experiences of participants attempting to independently exercise given the disruption of the programme, offering insights into transitioning out of the programme. Finally, we did not analyse interview data in relation to the HDQ scores, rather these scores were used to describe the study sample. As the HDQ is an outcome measure used within the PT-led exercise programme, future research may use the HDQ to measure change in disability with the exercise programme.

\section{CONCLUSIONS}

We described experiences engaging in a group-based PT-led exercise programme among PLWH prior-to, during and after the programme, as well as in anticipation of the transition to independent exercise. Interprofessional leaders, the HIV-specific facility, flexible schedule and social support positively influenced engagement. Perceived benefits in domains of health facilitated engagement and supported the transition to independent exercise, though some challenges remain. These challenges include pre-existing extrinsic barriers, such as financial inaccessibility and apprehension towards exercising alone. Results can inform future exercise programming for PLWH and other complex populations to facilitate and maintain engagement, and support successful transition to independent exercise.

\section{Author affiliations}

${ }^{1}$ Department of Physical Therapy, Temerty Faculty of Medicine, University of Toronto, Toronto, Ontario, Canada

${ }^{2}$ Casey House, Toronto, Ontario, Canada

${ }^{3}$ Department of Research Methods, Evidence, and Impact, McMaster University, Hamilton, Ontario, Canada

${ }^{4}$ Institute of Health Policy, Management and Evaluation (IHPME), University of Toronto, Toronto, Ontario, Canada

${ }^{5}$ Rehabilitation Sciences Institute (RSI), University of Toronto, Toronto, Ontario, Canada

\section{Twitter Kelly K. 0'Brien @kellyobrien25}

Acknowledgements This study was completed in partial fulfilment of the requirements for a Masters in Physical Therapy degree at the University of Toronto. The authors thank the participants of the group-based exercise programmes, our pilot interviewee, Greg Robinson for taking part in a pilot interview and proving feedback on the interview guide, and Casey House for their ongoing participation and collaboration. We also acknowledge Rachel Aubry (University of Toronto) for her assistance with participant recruitment.

Contributors KKO and SCC designed the study and provided guidance throughout the research process. KKO and SCC possess expertise in qualitative methodology and HIV and exercise research. KKO and SCC supervised TSH, AR, ZA-T, HB and SR (MScPT students) who developed the protocol, collected and analysed the data and drafted the manuscript in partial fulfilment of the requirements for a MSCPT degree at the University of Toronto. TSH, AR, ZA-T, HB and SR (MScPT students) developed skills in qualitative methodology by attending lectures; completing readings on qualitative methodology and study design; understanding steps of recruitment, data collection and analysis; completing a literature review' developing the research protocol, interview guide, demographic questionnaire, coding scheme and considering the ethical issues associated with this research. All steps were closely reviewed and guided by KKO and SCC. CM (Physiotherapist) and RW (Registered Nurse) contributed to participant recruitment and provided expert knowledge pertaining to the exercise programme. All authors read and approved the final manuscript.

Funding This research was supported by the Connaught Community Partner Research Programme (University of Toronto). KKO is supported by a Canada Research Chair in Episodic Disability and Rehabilitation from the Canada Research Chairs Program.

Competing interests None declared.

Patient consent for publication Obtained.

Ethics approval This study received approval from the University of Toronto Research Ethics Board (Protocol \#38411).

Provenance and peer review Not commissioned; externally peer reviewed.

Data availability statement All data relevant to the study are included in the article or uploaded as online supplemental information. Relevant data to this study are available in the article. No additional data are available in accordance with the protocol that was approved by the University of Toronto Health Sciences Research Ethics Board (Protocol \#: 38441).

Supplemental material This content has been supplied by the author(s). It has not been vetted by BMJ Publishing Group Limited (BMJ) and may not have been peer-reviewed. Any opinions or recommendations discussed are solely those of the author(s) and are not endorsed by BMJ. BMJ disclaims all liability and responsibility arising from any reliance placed on the content. Where the content includes any translated material, BMJ does not warrant the accuracy and reliability of the translations (including but not limited to local regulations, clinical guidelines, terminology, drug names and drug dosages), and is not responsible for any error and/or omissions arising from translation and adaptation or otherwise.

Open access This is an open access article distributed in accordance with the Creative Commons Attribution Non Commercial (CC BY-NC 4.0) license, which permits others to distribute, remix, adapt, build upon this work non-commercially, and license their derivative works on different terms, provided the original work is properly cited, appropriate credit is given, any changes made indicated, and the use is non-commercial. See: http://creativecommons.org/licenses/by-nc/4.0/.

\section{ORCID iDs}

Soo Chan Carusone http://orcid.org/0000-0003-3977-0523

Kelly K. O'Brien http://orcid.org/0000-0002-1632-6537

\section{REFERENCES}

1 Antiretroviral Therapy Cohort Collaboration. Survival of HIV-positive patients starting antiretroviral therapy between 1996 and 2013: a collaborative analysis of cohort studies. Lancet HIV 2017;4:e349-56.

2 Serrão R, Piñero C, Velez J, et al. Non-Aids-Related comorbidities in people living with HIV-1 aged 50 years and older: the aging positive study. Int J Infect Dis 2019;79:94-100.

3 Demir OM, Candilio L, Fuster D, et al. Cardiovascular disease burden among human immunodeficiency virus-infected individuals. Int $J$ Cardiol 2018;265:195-203.

4 Nansseu JR, Bigna JJ, Kaze AD, et al. Incidence and risk factors for prediabetes and diabetes mellitus among HIV-infected adults on antiretroviral therapy: a systematic review and meta-analysis. Epidemiology 2018;29:431-41.

5 De Francesco D, Sabin CA, Reiss P. Multimorbidity patterns in people with HIV. Curr Opin HIV AIDS 2020;15:110-7.

6 Valderas JM, Starfield B, Sibbald B, et al. Defining comorbidity: implications for understanding health and health services. Ann Fam Med 2009; 7:357-63.

7 Marengoni A, Angleman S, Melis R, et al. Aging with multimorbidity: a systematic review of the literature. Ageing Res Rev 2011;10:430-9.

8 Guaraldi G, Zona S, Brothers TD, et al. Aging with HIV vs. HIV seroconversion at older age: a diverse population with distinct comorbidity profiles. PLoS One 2015;10:e0118531. 
9 O'Brien KK, Bayoumi AM, Strike C, et al. Exploring disability from the perspective of adults living with HIV/AIDS: development of a conceptual framework. Health Qual Life Outcomes 2008;6:76.

10 Rusch M, Nixon S, Schilder A, et al. Impairments, activity limitations and participation restrictions: prevalence and associations among persons living with HIV/AIDS in British Columbia. Health Qual Life Outcomes 2004;2:46.

11 O'Brien KK, Davis AM, Strike C, et al. Putting episodic disability into context: a qualitative study exploring factors that influence disability experienced by adults living with HIV/AIDS. J Int AIDS Soc 2009:12:5.

12 O'Brien KK, Solomon P, Trentham B, et al. Evidence-Informed recommendations for rehabilitation with older adults living with HIV: a knowledge synthesis. BMJ Open 2014;4:e004692.

13 Dekker J, Buurman BM, van der Leeden M. Exercise in people with comorbidity or multimorbidity. Health Psychol 2019;38:822-30.

14 O'Brien KK, Tynan A-M, Nixon SA, et al. Effectiveness of progressive resistive exercise (pre) in the context of HIV: systematic review and meta-analysis using the Cochrane collaboration protocol. BMC Infect Dis 2017;17:268.

15 O'Brien KK, Tynan A-M, Nixon SA, et al. Effectiveness of aerobic exercise for adults living with HIV: systematic review and metaanalysis using the Cochrane collaboration protocol. BMC Infect Dis 2016;16:182

16 Nosrat S, Whitworth JW, Ciccolo JT. Exercise and mental health of people living with HIV: a systematic review. Chronic IIIn 2017:13:299-319.

17 Fillipas S, Bowtell-Harris CA, Oldmeadow LB, et al. Physical activity uptake in patients with HIV: who does how much? Int J STD AIDS 2008;19:514-8.

18 Vancampfort D, Mugisha J, De Hert M, et al. Global physical activity levels among people living with HIV: a systematic review and metaanalysis. Disabil Rehabil 2018;40:388-97.

19 Li A, McCabe T, Silverstein E, et al. Community-Based exercise in the context of HIV: factors to consider when developing and implementing community-based exercise programs for people living with HIV. J Int Assoc Provid AIDS Care 2017;16:267-75.

20 Simonik A, Vader K, Ellis D, et al. Are you ready? exploring readiness to engage in exercise among people living with HIV and multimorbidity in Toronto, Canada: a qualitative study. BMJ Open 2016;6:e010029.

21 Quigley A, Baxter L, Keeler L, et al. Using the theoretical domains framework to identify barriers and facilitators to exercise among older adults living with HIV. AIDS Care 2019;31:163-8.

22 Schaink AK, Kuluski K, Lyons RF, et al. A scoping review and thematic classification of patient complexity: offering a unifying framework. J Comorb 2012;2:1-9.

23 Hanson CL, Oliver EJ, Dodd-Reynolds CJ, et al. How do participant experiences and characteristics influence engagement in exercise referral? A qualitative longitudinal study of a scheme in Northumberland, UK. BMJ Open 2019;9:e024370.

24 Montgomery CA, Henning KJ, Kantarzhi SR, et al. Experiences participating in a community-based exercise programme from the perspective of people living with HIV: a qualitative study. BMJ Open 2017;7:e015861.

25 Salbach NM, Howe J-A, Brunton K, et al. Partnering to increase access to community exercise programs for people with stroke, acquired brain injury, and multiple sclerosis. J Phys Act Health 2014;11:838-45.

26 Stuart M, Benvenuti F, Macko R, et al. Community-Based adaptive physical activity program for chronic stroke: feasibility, safety, and efficacy of the Empoli model. Neurorehabil Neural Repair 2009;23:726-34.

27 O'Brien KK, Bayoumi AM, Solomon P, et al. Evaluating a community-based exercise intervention with adults living with HIV: protocol for an interrupted time series study. BMJ Open 2016;6:e013618.

28 Brown D, Claffey A, Harding R. Evaluation of a physiotherapy-led group rehabilitation intervention for adults living with HIV: referrals, adherence and outcomes. AIDS Care 2016;28:1495-505.

29 Fillipas S, Oldmeadow LB, Bailey MJ, et al. A six-month, supervised, aerobic and resistance exercise program improves self-efficacy in people with human immunodeficiency virus: a randomised controlled trial. Aust J Physiother 2006;52:185-90.

30 deBoer $\mathrm{H}$, Andrews M, Cudd S, et al. Where and how does physical therapy fit? integrating physical therapy into interprofessional HIV care. Disabil Rehabil 2019;41:1768-77.

31 Chung $\mathrm{CH}$, Lou VWQ. Effects of supervised exercise on physical health and quality of life among older adults living with HIV in Hong Kong. J Assoc Nurses AIDS Care 2020;31:353-8.
32 Cobbing S, Chetty V. Participants' reflections on a home-based rehabilitation intervention for people living with HIV in KwaZulu-Natal, South Africa. J Assoc Nurses AIDS Care 2019;30:218-23.

33 McDermott A, Zaporojan L, McNamara P, et al. The effects of a 16week aerobic exercise programme on cognitive function in people living with HIV. AIDS Care 2017;29:667-74.

34 Worthington C, Myers T, O'Brien K, et al. Rehabilitation in HIV/AIDS: development of an expanded conceptual framework. AIDS Patient Care STDS 2005;19:258-71.

35 Colorafi KJ, Evans B. Qualitative descriptive methods in health science research. HERD 2016;9:16-25.

36 Gough K, Karapita S. Facing the future together: an innovative response to the urgent HIVIAIDS crisis in Toronto. Toronto, ON: Casey House, 2011.

37 Borg G. Perceived exertion: a note on "history" and methods. Medicine and science in sports. Med Sci Sports 1973:5:90-3.

38 O'Brien KK, Solomon P, Ibáñez-Carrasco F, et al. Evolution of an international research collaborative in HIV and rehabilitation: community engaged process, lessons learned, and recommendations. Prog Community Health Partnersh 2018;12:395-408.

39 O'Brien KK, Ibáñez-Carrasco F, Solomon P. Research priorities for rehabilitation and aging with HIV: a framework from the CanadaInternational HIV and rehabilitation research collaborative (CIHRRC). AIDS Res Ther 2020;17.

40 Casey House Day Health Program. Casey house. Available: https:// www.caseyhouse.com/how-we-help/day-health-program-services [Accessed March 5, 2019].

41 Moser A, Korstjens I. Series: practical guidance to qualitative research. Part 3: sampling, data collection and analysis. Eur J Gen Pract 2018;24:9-18.

42 Phillippi J, Lauderdale J. A guide to field notes for qualitative research: context and conversation. Qual Health Res 2018;28:381-8.

43 O'Brien KK, Bayoumi AM, Stratford P, et al. Which dimensions of disability does the HIV disability questionnaire (HDQ) measure? a factor analysis. Disabil Rehabil 2015;37:1193-201.

44 O'Brien KK, Solomon P, Bergin C, et al. Reliability and validity of a new HIV-specific questionnaire with adults living with HIV in Canada and ireland: the HIV disability questionnaire (HDQ). Health Qual Life Outcomes 2015;13:124.

45 O'Brien KK, Solomon P, Bayoumi AM. Measuring disability experienced by adults living with HIV: assessing construct validity of the HIV disability questionnaire using confirmatory factor analysis. BMJ Open 2014;4:e005456.

46 O'Brien KK, Kietrys D, Galantino ML, et al. Reliability and validity of the HIV disability questionnaire (HDQ) with adults living with HIV in the United States. J Int Assoc Provid AIDS Care 2019;18:232595821 9888461:232595821988846

47 Brown DA, Simmons B, Boffito M, et al. Evaluation of the psychometric properties of the HIV disability questionnaire among adults living with HIV in the United Kingdom: a cross-sectional selfreport measurement study. PLoS One 2019;14:e0213222.

48 Braun V, Clarke V. Using thematic analysis in psychology. Qual Res Psychol 2006;3:77-101.

49 Flicker S, Nixon SA. The DEPICT model for participatory qualitative health promotion research analysis piloted in Canada, Zambia and South Africa. Health Promot Int 2015;30:616-24.

50 O'Brien K. Hiv disability questionnaire (HDQ) license agreement, administration and scoring manual, 2017.

51 Thomas SJ. Using web and paper questionnaires for data-based decision making. SAGE Publications, 2004: 131-61.

52 Caspersen CJ, Powell KE, Christenson GM. Physical activity, exercise, and physical fitness: definitions and distinctions for healthrelated research. Public Health Rep 1985;100:126-31.

53 Melin J, Nordin Åsa, Feldthusen C, et al. Goal-setting in physiotherapy: exploring a person-centered perspective. Physiother Theory Pract 2019:1-18.

54 Montoya JL, Jankowski CM, O'Brien KK, et al. Evidence-Informed practical recommendations for increasing physical activity among persons living with HIV. AIDS 2019;33:931-9.

55 Booth J, Moseley GL, Schiltenwolf M, et al. Exercise for chronic musculoskeletal pain: a biopsychosocial approach. Musculoskeletal Care 2017; 15:413-21.

56 Jones G, Hawkins K, Mullin R, et al. Understanding how adherence goals promote adherence behaviours: a repeated measure observational study with HIV seropositive patients. BMC Public Health 2012;12:587.

57 Filoramo MA. Improving goal setting and goal attainment in patients with chronic noncancer pain. Pain Manag Nurs 2007;8:96-101. 
58 Krasny-Pacini A, Hiebel J, Pauly F, et al. Goal attainment scaling in rehabilitation: a literature-based update. Ann Phys Rehabil Med 2013;56:212-30.

59 Malec JF. Goal attainment scaling in rehabilitation. Neuropsychol Rehabil 1999;9:253-75.

60 Cobbing S, Hanass-Hancock J, Deane M. Physiotherapy rehabilitation in the context of HIV and disability in KwaZulu-Natal, South Africa. Disabil Rehabil 2014;36:1687-94.

61 deBoer H, Cudd S, Andrews M, et al. Recommendations for integrating physiotherapy into an interprofessional outpatient care setting for people living with HIV: a qualitative study. BMJ Open 2019;9:e026827.

62 Dnes N, Coley B, Frisby K, et al. "A little bit of a guidance and a little bit of group support": a qualitative study of preferences, barriers, and facilitators to participating in community-based exercise opportunities among adults living with chronic pain. Disabil Rehabil 2020:1-10.

63 Reed M, Harrington R, Duggan A, et al. Meeting stroke survivors perceived needs: a qualitative study of a community-based exercise and education scheme. Clin Rehabil 2010;24:16-25.

64 Blank MB, Hanrahan NP, Fishbein M, et al. A randomized trial of a nursing intervention for HIV disease management among persons with serious mental illness. Psychiatr Serv 2011;62:1318-24.

65 Spirig R, Nicca D, Voggensperger J, et al. The advanced nursing practice team as a model for HIV/AIDS caregiving in Switzerland. $J$ Assoc Nurses AIDS Care 2004;15:47-55.

66 Watson S. HIV complex care and care coordination: the nurse's role. HIV Nursing 2018;18:38-46.

67 Merali S, Cameron JI, Barclay R, et al. Experiences of people with stroke and multiple sclerosis and caregivers of a community exercise programme involving a healthcare-recreation partnership. Disabil Rehabil 2020;42:1220-6.

$68 \mathrm{Li}$ X-M, Yuan X-Q, Wang J-J, et al. Evaluation of impact of social support and care on HIV-positive and AIDS individuals' quality of life: a nonrandomised community trial. J Clin Nurs 2017;26:369-78.

69 Moreno-Montoya J, Barragán AM, Martínez M, et al. Quality of life and perceived social support in people with HIV in Bogotá, Colombia. Biomedica 2018;38:577-85.

70 Matsumoto S, Yamaoka K, Takahashi K, et al. Social support as a key protective factor against depression in HIV-infected patients: report from large HIV clinics in Hanoi, Vietnam. Sci Rep 2017;7:15489.

71 Johs NA, Kellar-Guenther Y, Jankowski CM, et al. A qualitative focus group study of perceived barriers and benefits to exercise by selfdescribed exercise status among older adults living with HIV. BMJ Open 2019;9:e026294.

72 Ley C, Leach L, Barrio MR, et al. Effects of an exercise programme with people living with HIV: research in a disadvantaged setting. Afr $J$ AIDS Res 2014:13:313-9.

73 Gomes Neto M, Conceição CS, Oliveira Carvalho V, Carvalho $\mathrm{O} V$, et al. Effects of combined aerobic and resistance exercise on exercise capacity, muscle strength and quality of life in HIVinfected patients: a systematic review and meta-analysis. PLoS One 2015;10:e0138066.

74 Neff HA, Kellar-Guenther Y, Jankowski CM, et al. Turning disability into ability: barriers and facilitators to initiating and maintaining exercise among older men living with HIV. AIDS Care 2019;31:260-4.

75 Prochaska JO, Velicer WF. The transtheoretical model of health behavior change. Am J Health Promot 1997;12:38-48.

76 Mabweazara SZ, Leach LL, Ley C. Development of a contextsensitive physical activity intervention for persons living with HIV and AIDS of low socioeconomic status using the behaviour change wheel. BMC Public Health 2019;19:774.

77 Canadian Society for Exercise Physiology. Canadian 24-hour movement guidelines for adults ages 18-64 years: an integration of physical activity, sedentary behaviour, and sleep, 2011. Available: https://csepguidelines.ca/adults-18-64/ [Accessed 14 Jun 2021].

78 Vader K, Simonik A, Ellis D, et al. Perceptions of 'physical activity' and 'exercise' among people living with HIV: A qualitative study. Int $J$ Ther Rehabil 2017;24:473-82.

79 Mangona L, Daca T, Tchonga F, et al. Effect of different types of exercise in HIV + Mozambican women using antiretroviral therapy. Open AIDS J 2015;9:89-95.

80 Vader K, Carusone SC, Aubry R, et al. Strengths and challenges of implementing physiotherapy in an HIV community-based care setting: a qualitative study of perspectives of people living with HIV and healthcare providers. J Int Assoc Provid AIDS Care 2021;20:232595822110056. 